\title{
NUMERICAL SIMULATION OF THE AUTOGENOUS SHRINKAGE OF HARDENING PORTLAND CEMENT PASTE
}

\author{
P. Gao ${ }^{1,2 a}$, G. Ye ${ }^{2 b}$, J.X. Wei ${ }^{1 \mathrm{c}}$, Q.J. Y ${ }^{1 \mathrm{~d}}$ \\ ${ }^{1}$ School of Materials Science and Engineering, South China University of Technology \\ Guangzhou, 510640, People's Republic of China \\ ${ }^{2}$ Microlab, Faculty of Civil Engineering and Geosciences, Delft University of Technology \\ Delft, $2628 \mathrm{CN}$, The Netherlands \\ a.gao@tudelft.nl, ${ }^{\text {b g.ye@ @ tudelft.nl, }}$ jxwei@ scut.edu.cn, ${ }^{\mathrm{d}}$ concyuq@ scut.edu.cn,
}

Keywords: Autogenous Shrinkage, Numerical Simulation, Capillary Pressure.

\begin{abstract}
Autogenous shrinkage is the volume deformation of a closed, isothermal, cementitious material system not subjected to external forces. How to accurately predict the autogenous shrinkage in hardening cement-based materials is an important issue in concrete technology because autogenous shrinkage increases cracking risk and reduces the durability and service life of reinforced concrete structures. Many models simulated the autogenous shrinkage of cement-based materials based on some mechanisms such as capillary pressure and disjoining pressure. These models are normally empirical and cannot reveal the deformation behavior of cement-based materials under the internal load of the driving force of autogenous shrinkage in microscale. As a consequence, the reliability of these models are questionable.
\end{abstract}

This paper proposes a numerical model to simulate the autogenous shrinkage of hardening cement paste. A cement hydration and microstructure model HYMOSTRUC3D is used to simulate the microstructure of cement paste. A pore morphology based method is applied to describe the water and empty capillary pores distribution in the microstructure. Capillary pressure in the microstructure of cement paste is calculated from relative humidity measured by experiment based on Kelvin equation. A discrete algorithm is proposed to divide the hydration time into several static times. At each static time, a lattice finite element fracture method is used to simulate the deformation of simulated microstructure of cement paste under capillary pressure imposing. The autogenous shrinkage of hardening cement paste is equal to the sum of deformation of cement paste at each static time. The autogenous shrinkage of Portland cement paste with water to cement ratio of 0.3 is predicted. The simulation results are in a good agreement with experiments. 


\section{INTRODUCTION}

Autogenous shrinkage of cement-based materials (cement pastes, mortars and concretes, etc.) is defined as "the bulk deformation of a closed, isothermal, cementitious material system not subjected to external forces" [1]. Internal factors including the chemical shrinkage due to the cement hydration, the change of internal relative humidity caused by the consumption of water, and the pore structure of concrete are believed to result in autogenous shrinkage [2].

Autogenous shrinkage is harmful for concrete structures because it increases cracking risk and potentially reduces service life of concrete structures. Due to widespread usage of superplasticizers and high amount of supplementary cementitious materials (e.g. silica fume), modern concretes are characterized of low water to binder ratio (W/B) and fine pore structure, which significantly increases autogenous shrinkage [1-3].

Self-desiccation is considered as the main reason for autogenous shrinkage [1,2]. The products of cement hydration occupies less volume than the reactants during cement hydration. The volume difference between products and reactants is defined as chemical shrinkage [2]. Under sealed-conditions, chemical shrinkage causes "empty spaces" in the pore structure of cement-based materials, because the eternal water is not available to fill the volume difference due to cement hydration [3]. These "empty spaces" is then filled with vapour evaporated from water. After that, surface tension forms in the interface of gas phase (vapour) and liquid phase (water). Due to the effect of surface tension, meniscus forms $[2,3]$. Relative humidity (RH) in the vapour decreases at the same time due to meniscus formation. Thus this process is called self-desiccation. It is believed that self-desiccation rises internal tensile stresses in pore solution, which is considered as the driving force of autogenous shrinkage $[2,3]$.

Intensive studies have investigated how does self-desiccation rise internal tensile stresses. Three fundamental mechanisms were proposed: surface tension mechanism, capillary pressure mechanism, and disjoining pressure mechanism [4-9]. Based on these mechanisms, some numerical models $[4,7,8]$ were established to predict autogenous shrinkage. For example, Lura et al. [4] modelled the deformation of cement paste by a semi-macroscopic equation:

$$
\varepsilon_{L I N}=\frac{S \sigma_{c a p}}{3}\left(\frac{1}{K}-\frac{1}{K_{S}}\right)
$$

where $S$ is saturation fraction (-), $\sigma_{\text {cap }}$ is the capillary pressure in the pore fluid (MPa), K is bulk modulus of the whole porous body $(\mathrm{MPa})$ and $\mathrm{K}_{\mathrm{S}}$ is bulk modulus of the solid material (MPa).

Hua et al. also proposed the models for predicting autogenous shrinkage at macroscopic scale [7] and the scale of hydrating grains [8]. Capillary pressure was considered as the driving force of autogenous shrinkage in both scales. At macroscopic scale, an empirical equation was adopted to calculate deformation [7], while at the scale of hydrating grains an finite element approach was applied to simulate the shrinkage of a hydrating grain [8].

Although these models can predict the macroscopic linear or volume deformation of cement-based materials, however, they cannot illustrate neither the impose process of internal tensile stresses on the solid skeleton of cement paste nor the corresponding deformation of solid skeleton in microscale. Solid skeleton is defined as the solid phase of cement paste that provides the mechanical strength of cement paste.

This paper aims to propose a numerical model to simulate the autogenous shrinkage of cement paste in microscale. In this model, both the impose process of internal tensile stress and the deformation of solid skeleton in microscale are simulated. Internal capillary pressure 
is considered as the driving force of autogenous shrinkage. The numerical cement hydration and microstructure model , HYMOSTRUC3D [10-12] is used to simulate the microstructure of cement paste. The pore morphology-based method [13] is employed to distribute the water and empty capillary pore in the pore space. A discrete algorithm is proposed to divided the hydration time into several static times. At each static time, the lattice finite element fracture method [14] is adapted to predict the deformation of solid skeleton of cement paste under the load of internal capillary pressure. The autogenous shrinkage of hardening cement paste is equal to the sum of the deformation of cement paste at each static time.

\section{MODELLING APPROACH}

Internal capillary pressure is considered as the driving force of autogenous shrinkage in this simulation. The outline of the simulation process is shown in Figure 1. The simulation process can be divided into three stages. In stage 1, HYMOSTRUC3D [10-12] is used to obtain the degree of hydration and the microstructure of cement paste. In stage 2, the water and empty capillary pores distribution in the simulated pore structure of cement paste is modelled. In stage 3, the lattice finite element fracture method is applied to simulate the deformation of skeleton phase of cement paste under the impose of capillary pressure calculated from measured RH.

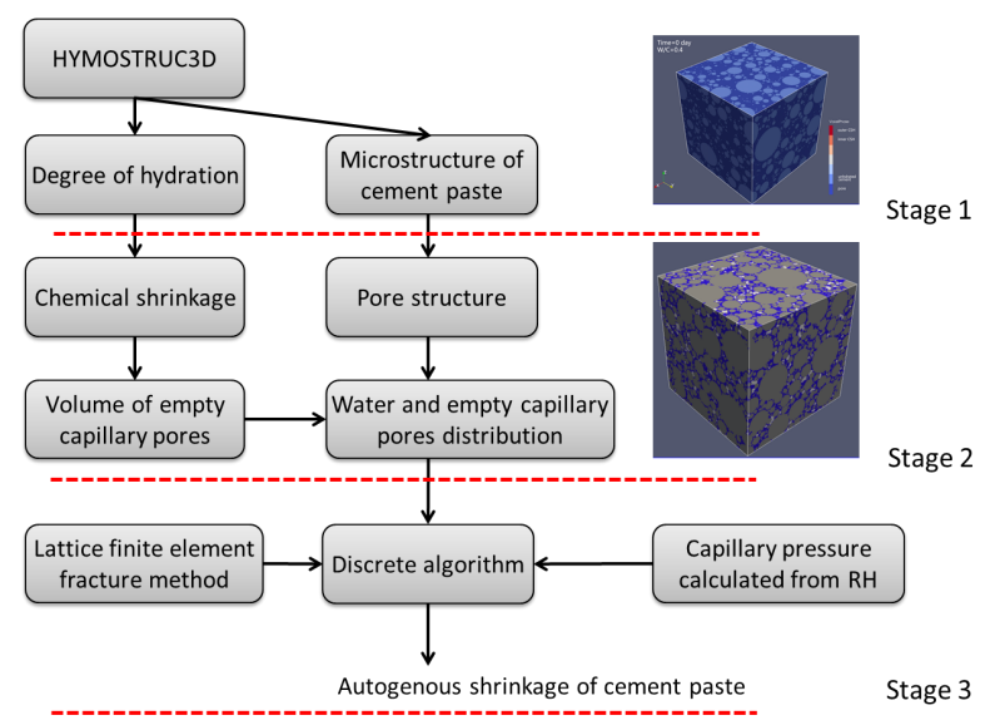

Figure 1 Simulation process of autogenous shrinkage of cement paste

\subsection{Hydration and microstructure of Portland cement}

HYMOSTRUC3D is a numerical model that can simulate the hydration and microstructure of Portland cement paste [10-12]. The numerical microstructure outputted by HYMOSTRUC3D contains several phases of Portland cement paste, including capillary pores, unhydrated cement, inner C-S-H, outer C-S-H, and calcium hydroxide $(\mathrm{CH})$. For example, Figure 2 shows the simulated microstructure of Portland cement paste with a water to cement ratio (W/C) of 0.3 at the age of 7 days. The main input parameters of HYMOSTRUC3D include mineral composition of cement, particle size distribution of cement, W/C, and model parameters like penetration rate $\left(\mathrm{K}_{0}\right)$ and transition thickness $\left(\delta_{\mathrm{tr}}\right)$ of cement. More information about the input parameters and model parameters of HYMOSTRUC3D can be referred to [10-12]. 
In this simulation, the mineral composition of Portland cement is: $\mathrm{C}_{3} \mathrm{~S}(62.0 \%), \mathrm{C}_{2} \mathrm{~S}$ (10.5\%), $\mathrm{C}_{3} \mathrm{~A}$ (7.3\%), $\mathrm{C}_{4} \mathrm{AF}(10.2 \%)$ [12]. The particle size distribution of Portland cement is shown in Figure 3 [12]. The W/C of cement paste is 0.3. As indicated in Table 1, the model parameters $\mathrm{K}_{0}$ and $\delta_{\text {tr }}$ are calculated from the mineral composition of Portland cement based on the method described by Tuan [15].

Table 1 Two model parameters $\mathrm{K}_{0}$ and $\delta_{\text {tr }}$ of different cement minerals [15]

\begin{tabular}{lll}
\hline Phase & $\mathrm{K}_{0}(\mu \mathrm{m} /$ hour $)$ & $\delta_{\text {tr }}(\mu \mathrm{m})$ \\
\hline $\mathrm{C}_{3} \mathrm{~S}$ & $0.0635+0.0195 \times\left(1-\% \mathrm{C}_{3} \mathrm{~S}\right)$ & $2.1199+1.4707 \times\left(1-\% \mathrm{C}_{3} \mathrm{~S}\right)$ \\
$\mathrm{C}_{2} \mathrm{~S}$ & $0.0033+0.0020 \times\left(1-\% \mathrm{C}_{2} \mathrm{~S}\right)$ & $2.0730+1.1528 \times\left(1-\% \mathrm{C}_{2} \mathrm{~S}\right)$ \\
$\mathrm{C}_{3} \mathrm{~A}$ & $1.2118-1.1714 \times\left(1-\% \mathrm{C}_{3} \mathrm{~A}\right)$ & $2.3280+1.2758 \times\left(1-\% \mathrm{C}_{3} \mathrm{~A}\right)$ \\
$\mathrm{C}_{4} \mathrm{AF}$ & 0.02 & 1.19 \\
\hline
\end{tabular}

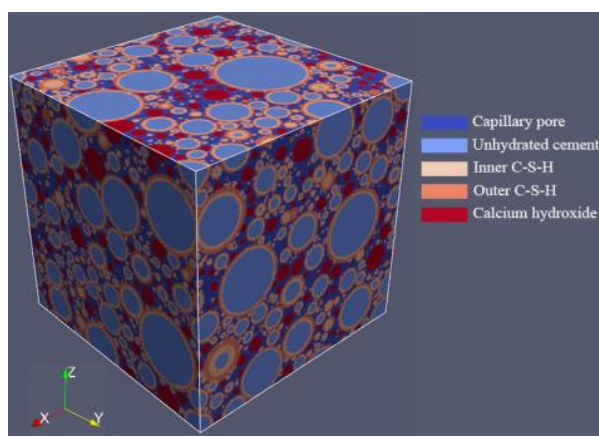

Figure 2 Simulated microstructure of Portland cement paste by extended HYMOSTRUC3D $(100 \times 100 \times 100$ $\mu \mathrm{m}^{3}, \mathrm{~W} / \mathrm{C}=0.3,1$ day)

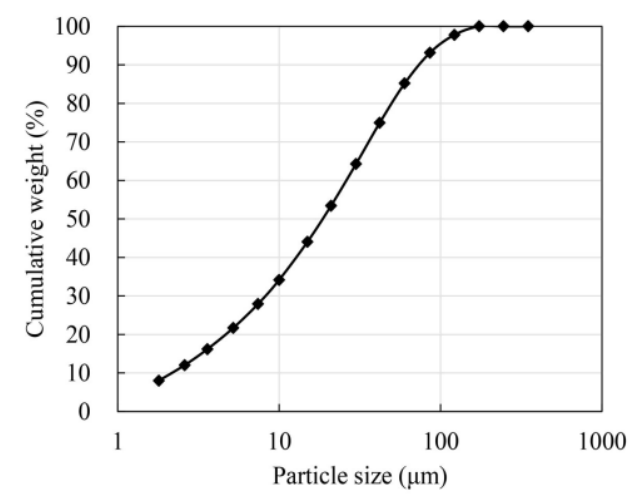

Figure 3 Particle size distribution of Portland cement

\subsection{Water and empty capillary pores distribution}

Because the products of cement hydration occupies less volume than the reactants of cement hydration (cement and water) some space that is not filled with water in the pore structure will be formed. The water free pace is defined as empty capillary pores. In this study we assume the pore space of cement paste contains two phases: water and empty capillary pores. The capillary pressure only exists in the liquid phase after the distribution of water and empty capillary pores is obtained. Therefore, the simulation of water and empty capillary pores is an necessary step for the simulation of the impose process of capillary pressure on the solid skeleton.

Water and empty capillary pores distribution can be simulated by pore-morphology based method established by Hilpert and Miller [13]. This method is commonly used to determine 
the capillary pressure-saturation curves for different porous media $[13,16]$. In this study the input parameters of pore-morphology based method are the volume of empty capillary pores and the pore structure of cement paste. The volume of empty capillary pores equals to:

$$
\mathrm{V}_{\text {Empty capillary pores }}=\mathrm{V}_{\text {Chemical shrinkage }} / \mathrm{V}_{\text {cement paste }}
$$

where $\mathrm{V}_{\text {Empty capillary pores }}$ is the volume of empty capillary pore $(\mathrm{ml} / \mathrm{ml})$. $\mathrm{V}_{\text {Chemical shrinkage }}$ is the volume of chemical shrinkage $(\mathrm{ml} / \mathrm{g})$. It can be simulated by HYMOSTRUC3D. $\mathrm{V}_{\text {cement paste }}$ is the volume of cement paste $(\mathrm{ml} / \mathrm{g})$.

The pore structure of cement paste is obtained by HYMOTRUC3D.

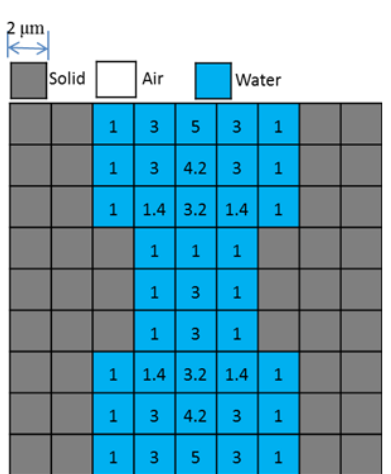

(a)

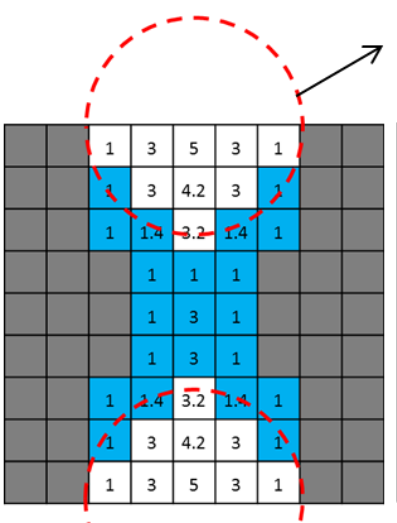

(b)

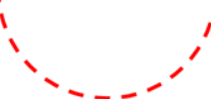

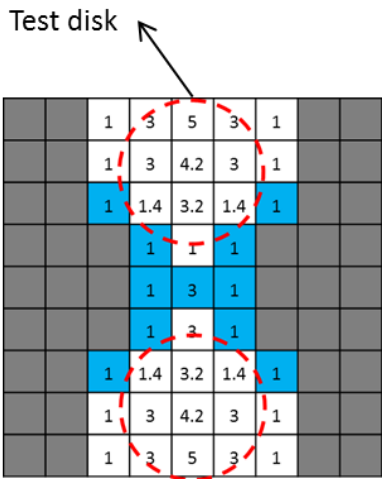

(c)

Figure 4 Schematic diagram of modelling self-desiccation process in cement paste based on poremorphology based method (In 2D). Grey is solid, blue is water, and white is empty capillary pore. Note: Sphere is used to replace disk in the algorithm for 3D. The size of voxel in the 3D simulation is $1 \mu \mathrm{m}$.

(1) Figure out the distance map of pore space.

(2) All the voxels that belong to disk $(\mathrm{R}=5 \mu \mathrm{m})$ are labelled as empty capillary pore.

(3) In the next step, all the voxels that belong to disk $(\mathrm{R}=3.2 \mu \mathrm{m})$ are labelled as empty capillary pore. After this step, the radii of disk changes to $3 \mu \mathrm{m}$. The "drying" process continues until the volume of empty voxels is equal to that of the empty capillary pores.

The schematic diagram for simulating the distribution water and empty capillary pores is shown in Figure 4. The algorithm contains the following steps:

(1) The simulated microstructure is digitalized into small voxels with identical size. These voxels represent solid and pore space (Figure 4).

(2) Then the distance map of pore space is figured out. In the distance map, each voxel in pore space has a distance value. This distance value is defined as the shortest distance between the centre of this pore voxel to the solid phase [17]. Figure 4a shows the schematic diagram of a distance map. Besides, all the pore voxels are labelled as water in this step (Figure 4a).

(3) A test disk (in 2D) or sphere (in 3D) is defined to determine which pore voxels should be empty. The radii of test disk or sphere is initially equal to the largest distance value in the distance map. For each pore voxel with largest distance value, a test disk or sphere is set up, and the centre of test disk or sphere is fixed at the centre of this pore voxel. After that, all the pore voxels in the range of test disks or spheres are labelled as empty capillary pores. For the example in Figure 4b, because only two pore voxels with largest pore distance exist, two test disks with radii of $5 \mu \mathrm{m}$ are given. The centres of the test disks are fixed at the centres of the pore voxels with the distance value of $5 \mu \mathrm{m}$, respectively. Then, all the pore voxels in the range of test disks are labelled as empty capillary pores. 
(4) In the next step, the radii of test disk or sphere changes to be second largest distance value. Similarly, for each pore voxels with this distance value, a test disk or sphere is set up, and the centre of test disk or sphere is fixed at the centre of this pore voxel. Then, all the pore voxels in the range of test disks or spheres are labelled as empty capillary pores. For the example shown in Figure 4c, there are two test disks with the radii of $4.2 \mu \mathrm{m}$, because the number of the voxels with the radii of $4.2 \mu \mathrm{m}$ is two. The centres of the test disks are fixed at the centres of the pore voxels with the distance value of $4.2 \mu \mathrm{m}$, respectively. All voxels in the range of test disks are labelled as empty capillary pores.

The iteration process described in (3) and (4) continues until the volume of total empty voxels are equal to that of empty capillary pores.

\subsection{Autogenous shrinkage of cement paste}

In stage 3 of Figure 1, a discrete algorithm is proposed to divide the hydration time into several static times. At each static time, a lattice finite element fracture analysis method is applied to simulate the impose process of capillary pressure on the solid skeleton of cement paste. The deformation of cement paste in microscale is then obtained. The autogenous shrinkage of hardening cement paste is equal to the sum of the deformation of cement paste at each static time. The model process are described as follow.

\section{(1) Lattice finite element fracture analysis method}

Lattice model has been used to simulate the fracture process of cement-based materials for decades [14]. Based on lattice model, Qian et al. [14] proposed a 3D lattice finite element fracture analysis to simulate the fracture process of cement paste. A GLAK (Generalized Lattice Analysis Kernel) package was developed to simulate the deformation of lattice mesh and the fracture process [14]. The main input files of GLAK package include lattice mesh and impose load. In this simulation the GLAK package is applied to simulate the impose of internal capillary pressure on the solid skeleton of cement paste and the corresponding deformation. The lattice mesh for GLAK package is generated from the microstructure of cement paste simulated by HYMOSTRUC3D. The internal capillary pressure, which is calculated from RH based on Kelvin equation, is considered as the impose load.

\section{(2) Lattice mesh construction}

The lattice mesh for GLAK package contains two fundamental parameters: lattice nodes and lattice beams [14]. Based on the method described in [14], this paper generates lattice mesh from the microstructure of cement paste simulated by HYMOSTRUC3D. The simulated microstructure of cement paste (Figure 5a and b) are classed as three phases (Figure 5c): solid phase (cement, inner product and outer product and $\mathrm{CH}$ indicated as grey), liquid phase (water indicated as blue), and vapor phase (empty capillary pore indicated as white). The lattice nodes are then randomly generated in each solid voxel. After that, beams are generated by connecting neighbor nodes (Figure 5d). The resolution of lattice mesh is $2 \mu \mathrm{m}$.

Table 2: Mechanical properties of different phases in microstructure (modified from [14])

\begin{tabular}{lll}
\hline Node type & Young's modulus $(\mathrm{GPa})$ & Shear modulus $(\mathrm{GPa})$ \\
\hline Cement & 135 & 52 \\
Inner product & 30 & 12 \\
Outer product & 22 & 8.9 \\
$\mathrm{CH}$ & 30 & 12 \\
\hline
\end{tabular}


The mechanical properties of beams are obtained according to the method described by Qian et al. [14]. Since each beam in the lattice mesh connects two nodes, the mechanical properties of each beam is assumed to be the average of that of two nodes.

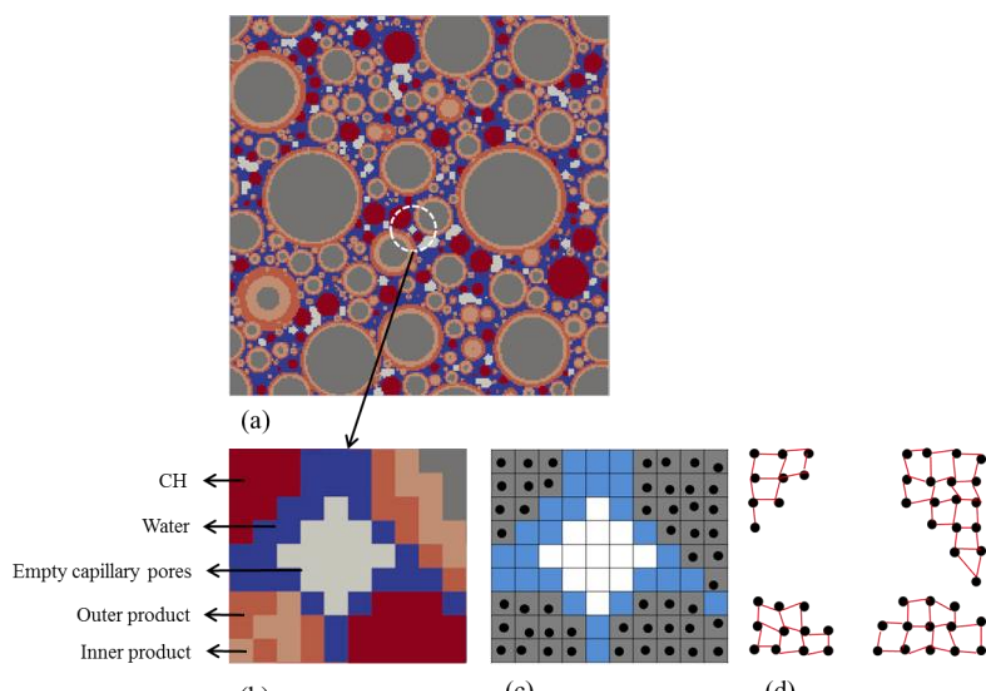

(b)

(c)

(d)

Figure 5 Schematic diagram of the construction of lattice mesh

\section{(3) Impose of capillary pressure}

During self-desiccation process, meniscus forms on the interfaces of water and vapour along with the formation of empty capillary pores. Due to meniscus formation, capillary pressure arises in the liquid and becomes an negative force that imposes on the solid skeleton.

In this simulation, the capillary pressure is assumed to be the same in any place of liquid. The capillary pressure can be calculated from $\mathrm{RH}[4]$ :

$$
\Delta \mathrm{p}=\frac{2 \gamma}{\mathrm{r}}=\frac{\operatorname{lnRH_{\mathrm {m}}} \times \mathrm{RT}}{\mathrm{V}_{\mathrm{m}}}
$$

where $\Delta \mathrm{p}$ is capillary pressure, $\gamma$ is surface tension, $\mathrm{r}$ is radii of pore, $\mathrm{RH}_{\mathrm{m}}$ is modified $\mathrm{RH}$ based on the method described in [4]. RH can be experimentally tested [4]. $\mathrm{R}$ is the universal gas constant, $\mathrm{T}$ is temperature, $\mathrm{V}_{\mathrm{m}}$ is the molar volume of the water.

The modified RH can be calculated by [4]:

$$
\mathrm{RH}_{\mathrm{m}}=\frac{\mathrm{RH}}{\mathrm{RH}_{\mathrm{s}}}
$$

Because the salt concentration in the pore solution of cement paste normally changes with cement hydration, $\mathrm{RH}_{\mathrm{S}}$ should be a function of hydration time. In this simulation, $\mathrm{RH}_{\mathrm{S}}$ is simplified as a constant $(98.2 \%)$ based on the report [4].

The capillary force imposed on the node is calculated by:

$$
\mathrm{F}_{\text {capillary }}=\Delta \mathrm{p} \times \mathrm{S}
$$

where $S$ is the area of the node. It depends on the resolution of lattice mesh.

The direction of capillary force on each node is figured out according to the rule: only the nodes that contact with liquid phase are imposed capillary force. For instance, the direction of capillary force on each node in Figure $5 d$ is indicated in Figure 6. 


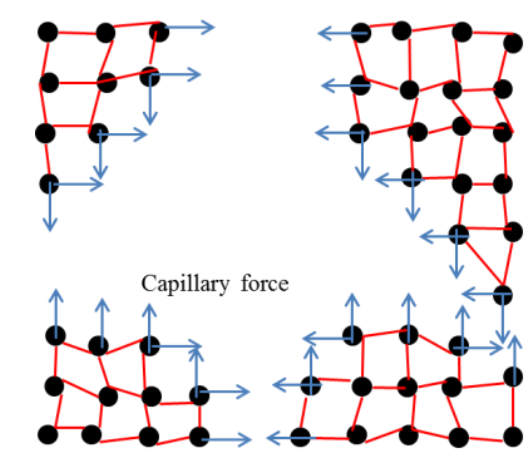

Figure 6 Impose of capillary pressure on lattice

\section{(4) Discrete algorithm for calculating autogenous shrinkage of hardening cement paste}

The above steps can calculate the deformation of cement paste at a static time. However, because both the microstructure of hardening cement paste and the capillary pressure in the pore solution change with the hydration time, the autogenous shrinkage of hardening cement paste is not static. We propose a discrete algorithm to calculate dynamic process of the autogenous shrinkage of hardening cement paste.

The schematic diagram of discrete algorithm for calculating autogenous shrinkage of hardening cement paste is shown in Figure 7. We assume at time $i$, the imposed force is $F_{i}$ and the length of specimen is $\mathrm{L}_{\mathrm{i}}$ after imposing. In the next time, new hydration products are generated in the microstructure, and the volume of solid phase increases due to cement hydration. Thus, the lattice mesh of microstructure at this time contains more nodes and beams than that at the last time. The increased nodes and beams are defined as new nodes and new beams (Figure $7 b$ ). The imposed force becomes to $F_{i+1}$. The extra force is defined as $\Delta \mathrm{F}_{\mathrm{i}+1}$, being equal to $\mathrm{F}_{\mathrm{i}+1}-\mathrm{F}_{\mathrm{i}}$. After loading this extra force, an extra strain $\left(\Delta \varepsilon_{\mathrm{i}+1}\right)$ is formed. Then, the length of specimen $\left(\mathrm{L}_{\mathrm{i}+1}\right)$ at time $\mathrm{i}+1$ after loading extra force is equal to:

$$
\mathrm{L}_{\mathrm{i}+1}=\left(1-\Delta \varepsilon_{\mathrm{i}+1}\right) \times \mathrm{L}_{\mathrm{i}}
$$
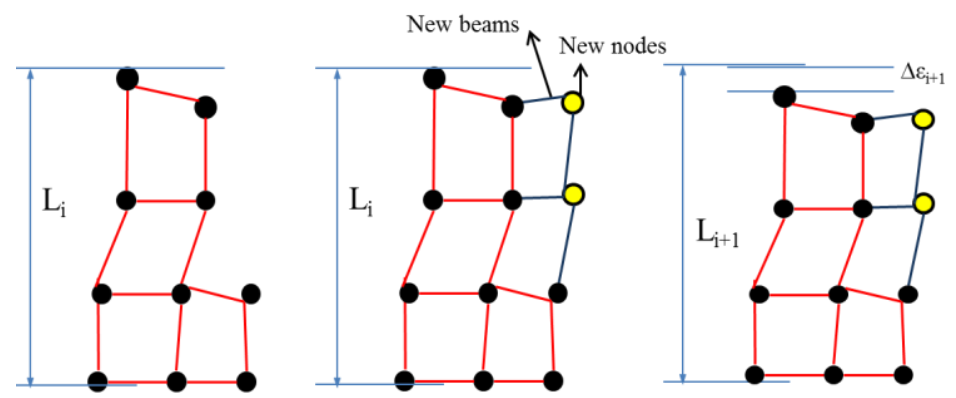

(a) After loading $F_{i}$ at time i. (b) Before loading $\Delta F_{i+1}$ at time i+1. (c) After loading $\Delta F_{i+1}$ at time $i+1$ Figure 7 Schematic diagram of discrete algorithm for calculating autogenous shrinkage of hardening cement paste

The autogenous shrinkage of cement paste at time $\mathrm{n}$ is calculated by:

$$
\mathrm{L}_{\mathrm{n}}=\left(1-\Delta \varepsilon_{\mathrm{n}}\right) \times\left(1-\Delta \varepsilon_{\mathrm{n}-1}\right) \cdots\left(1-\Delta \varepsilon_{1}\right) \mathrm{L}_{0}
$$

where $\mathrm{L}_{0}$ is the length of specimen at time zero.

The volume shrinkage of specimen is calculated by:

$$
\mathrm{V}_{\text {Shrinkage }}=1-\left(1-\mathrm{L}_{\mathrm{n}} / \mathrm{L}_{0}\right)^{3}
$$


In the discrete algorithm, the hydration time (from time zero to final testing time) is divided into several static times. The interval between two static times is not identical. Since cement commonly hydrates more quickly at early age than at later age, the interval is short at early age, and becomes long with increasing age. In this simulation, the period from time zero (5 hours after mixing) up to 7 days is divided into 37 static times (Figure 8).

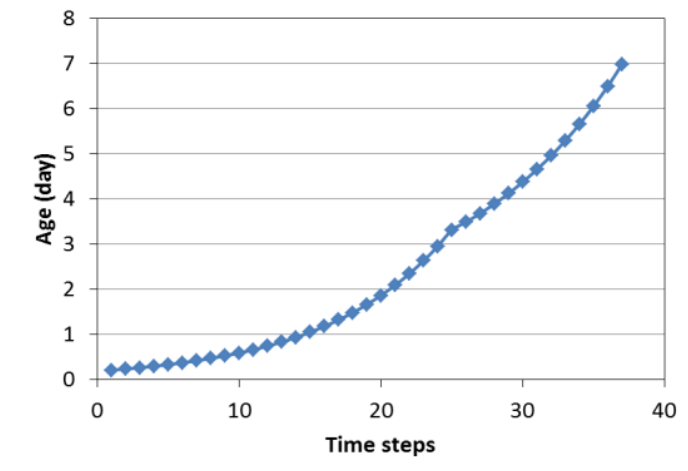

Figure 8 Time steps in the discrete algorithm

\section{SIMULATION RESULTS AND DISCUSSION}

\subsection{Hydration and microstructure}

The simulated degree of hydration of Portland cement paste with W/C of 0.3 is shown in Figure 9. The value is in a good agreement with experiment obtained by non-evaporable water method [12].

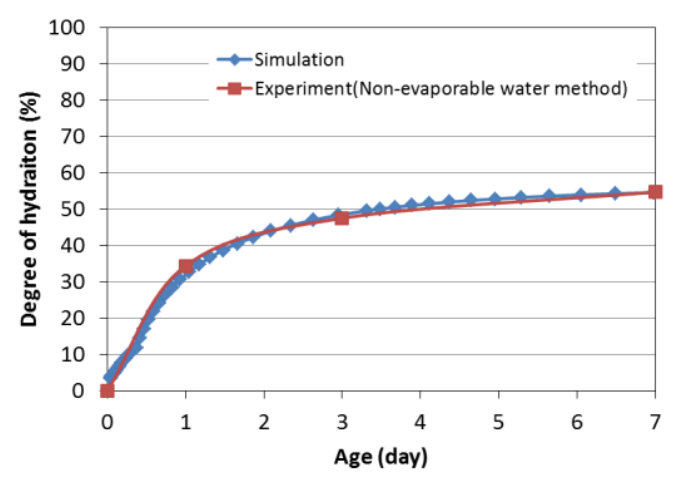

Figure 9 Simulated and experimental measured degree of hydration of cement paste

The simulated microstructures of Portland cement paste are show in Figure 10. The solid skeletons of cement paste are obtained. 

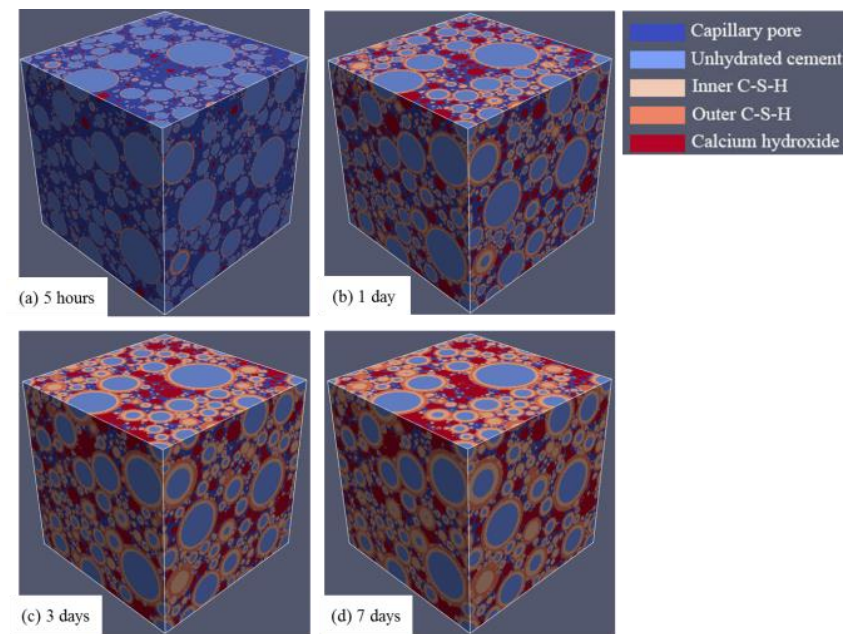

Figure 10 Simulated microstructures of cement pastes up to 7 days $\left(100 \times 100 \times 100 \mu \mathrm{m}^{3}\right)$

\subsection{Water and empty capillary pores distribution}

The simulated water and empty capillary pores distribution is shown in Figure 11. It is clear that the empty capillary pores are firstly formed in "big" pore space.
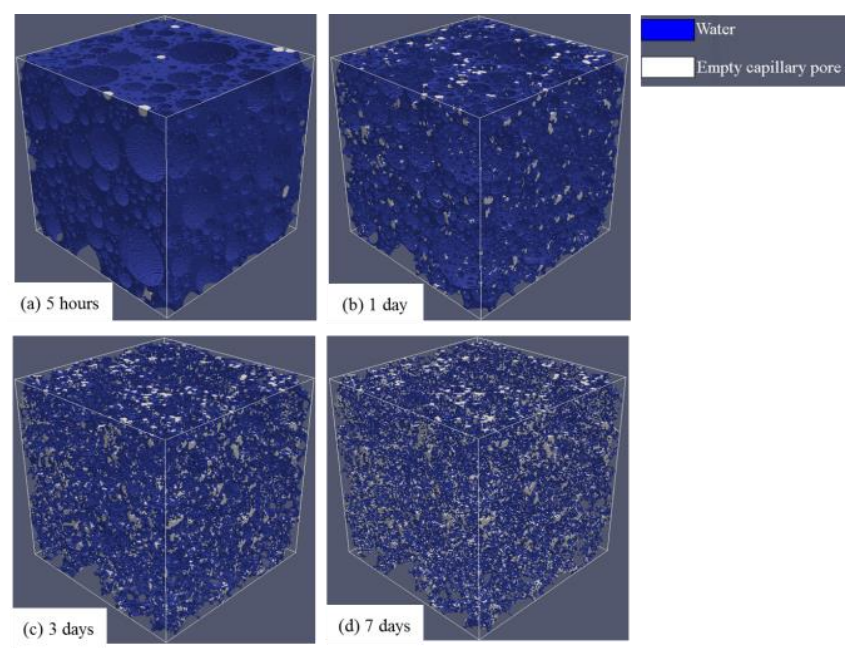

Figure 11 Simulated water and empty capillary pores distribution of cement pastes up to 7 days $\left(100 \times 100 \times 100 \mu \mathrm{m}^{3}\right)$

\subsection{Autogenous shrinkage of hardening cement paste}

The RH used in the simulation is obtained by the experimental study from $\mathrm{Lu}$ and van Breugel [18]. The results are shown in Figure 12a. After modification by using Eq. 4, RH is increased (red line in Figure 12a). The capillary pressure is calculated based on Kelvin equation (Eq.3) and shown in Figure 12b. 


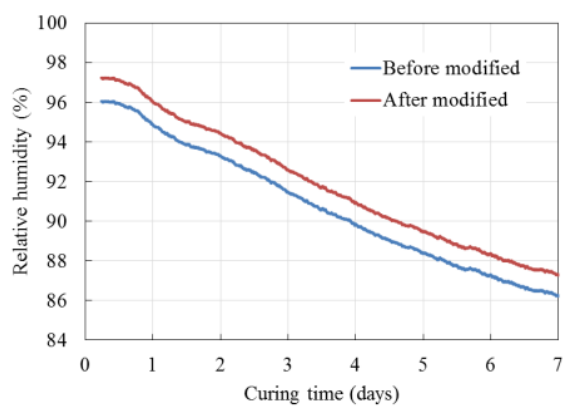

(a) $\mathrm{RH}$

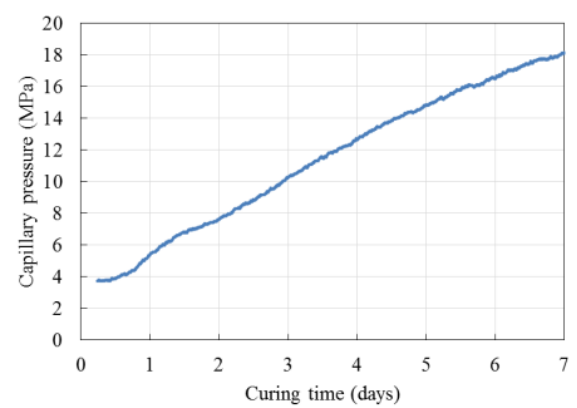

(b) Capillary pressure

Figure 12 Relative humidity (measured and modified by Eq. 4) and capillary pressure used in the simulation

The deformation of solid skeleton due to capillary pore is indicated in Figure 13. It illustrates that the solid skeleton shrinks due to the effect of capillary pressure (Figure 13b). The simulation results indicate that the surface of solid skeleton after shrinking is not smooth. This is because the beams have different mechanical properties. The mechanical properties of blue beams are higher than that of red beams, because blue beams consist of inner product and unhydrated cement, while red beams consist of outer product. In consequence, the deformation is not homogenous.

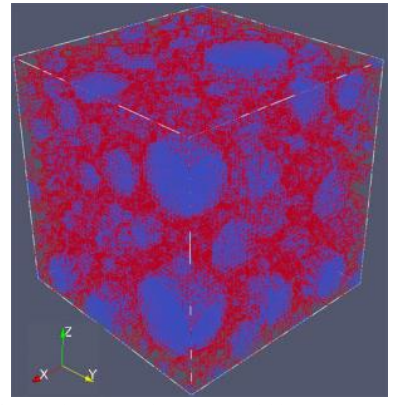

(a) Before imposing capillary pressure

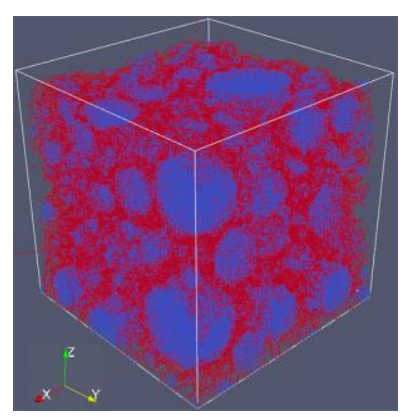

(b) After imposing capillary pressure

Figure 13 Simulated deformation of solid skeleton due to capillary pressure effect. $\left(100 \times 100 \times 100 \mu \mathrm{m}^{3}\right.$, 1 day) Note: the deformation is magnified for the purpose of visualization. Red beams are imposed load, and blue beam are not imposed load.

The simulated volume deformation of cement paste is compared with experimental data measured by $\mathrm{Lu}$ and van Breugel [18]. Lu and van Breugel used corrugated tube method to determine the autogenous shrinkage of Portland cement with a W/C of 0.3. Final setting time was adopted as time zero. The results of autogenous shrinkage of cement paste are shown in Figure 14. It is observed that the simulation result fits the experiment curve well.

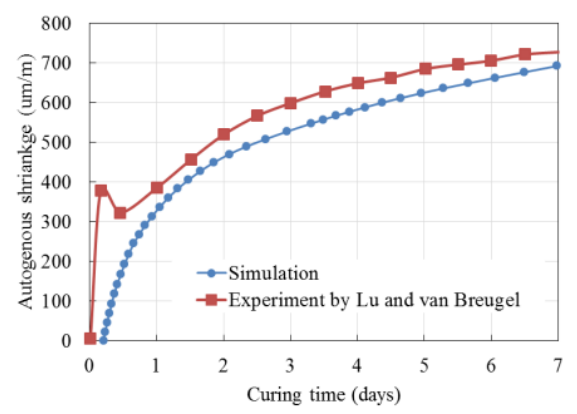

Figure 14 Autogenous shrinkage of cement paste 


\section{CONCLUSIONS}

This paper proposes a numerical model to predict the autogenous shrinkage of hardening Portland cement paste. Capillary pressure is considered as the driving force for autogenous shrinkage. A discrete algorithm is proposed to calculate the autogenous shrinkage of hardening cement paste. In the discrete algorithm, the hydration time from 5 hours to 7 days is divided into 37 static times. For each static time, the lattice finite element fracture analysis method is employed to simulate the impose of increased capillary pressure on solid skeleton of cement paste. The dynamic autogenous shrinkage is equal to the sum of the deformation of cement paste at each step. The simulated autogenous shrinkage of hardening Portland cement paste $(\mathrm{W} / \mathrm{C}=0.3)$ is in a good agreement with that measured from experiment.

\section{ACKNOWLEDGEMENTS}

This work was funded by the China Scholarship Council (CSC), the Research Centre of TU Delft in Urban System and Environment (No. C36103) and the National Basic Research Program of China (973 Program: 2011CB013800).

\section{REFERENCES}

[1] O.M. Jensen, P.F. Hansen, Autogenous deformation and RH-change in perspective. Cement and Concrete Research, 31, 1859-1865, 2001.

[2] D.P. Bentz, O.M. Jensen, Mitigation strategies for autogenous shrinkage cracking. Cement and Concrete Composites, 26, 677-685, 2004.

[3] D.P. Bentz, A review of early-age properties of cement-based materials. Cement and Concrete Research, 31, 1859-1865, 2008.

[4] P. Lura, O.M. Jensen, K. van Breugel, Autogenous shrinkage in high-performance cement paste: An evaluation. Cement and Concrete Research, 33, 913-922, 2003.

[5] K. van Breugel, Numerical modelling of volume changes at early ages-Potential, pitfalls and challenges. Materials and Structures, 34, 293-301, 2001.

[6] F.H. Wittmann, Surface tension skrinkage and strength of hardened cement paste. Matériaux et Construction. 1, 547-552, 1968.

[7] C. Hua, P. Acker, A. Ehrlacher, Analyses and models of the autogenous shrinkage of hardening cement paste: I. Modelling at macroscopic scale. Cement and Concrete research, 25, 1457-1468, 1995.

[8] C. Hua, A. Ehrlacher, P. Acker, Analyses and models of the autogenous shrinkage of hardening cement paste II. Modelling at scale of hydrating grains. Cement and concrete research, 27, 245-258, 1997.

[9] F. Beltzung, F.H. Wittmann, Role of disjoining pressure in cement based materials. Cement and Concrete Research, 35, 2364-2370, 2005.

[10] K. van Breugel, Simulation of hydration and formation of structure in hardening cement-based materials, PhD Thesis, Delft, Delft University of Technology, The Netherlands, 1991. 
[11] E.A.B. Koenders, Simulation of volume changes in hardening cement-based materials, PhD Thesis, Delft University of Technology, Delft, The Netherlands, 1997.

[12] G. Ye, Experimental Study and Numerical Simulation of the Development of the microstructure and permeability of cementitious materials, PhD Thesis, Delft, Delft University of Technology, The Netherlands, 2003.

[13] M. Hilpert, and C.T. Miller, Pore-morphology-based simulation of drainage in totally wetting porous media. Advances in Water Resources, 24, 243-255, 2001.

[14] Z.W. Qian, E. Schlangen, G. Ye, K. van Breugel, Prediction of mechanical properties of cement paste at microscale. Materiales de Construccion, 60, 7-18, 2010.

[15] Tuan N.V., Rice husk ash as a mineral admixture for ultra high performance concrete. PhD Thesis, Delft, Delft University of Technology, The Netherlands, 2011.

[16] V.P. Schulz, E.A. Wargo, E.C. Kumbur, Pore-Morphology-Based Simulation of Drainage in Porous Media Featuring a Locally Variable Contact Angle. Transport in Porous Media, 107, 13-25, 2015.

[17] E. Vergés, D. Ayala, S. Grau, D. Tost, 3D reconstruction and quantification of porous structures. Computers and Graphics, 32, 438-444, 2008.

[18] T.S. Lu, K. van Bregugel, Analyzing and modelling time dependent-component of autogenous shrinkage of hardening cement paste and mortar. $14^{\text {th }}$ International Congress on the Chemistry of Cement, Beijing, China, October 13-16, 2015 